\title{
Neutrino-induced Nucleosynthesis in Core-Collapse Supernovae
}

\author{
D.H. Hartmann ${ }^{1}$, W.C. Haxton ${ }^{2}$, R.D. Hoffman ${ }^{3}$, and S.E. Woosley ${ }^{1,3}$ \\ ${ }^{1}$ Physics Department, University of California. \\ Lawrence Livermore National Laboratory \\ ${ }^{2}$ Nuclear Theory Group, Department of Physics, FM-15 \\ University of Washington, Seattle, Washington 98195 \\ ${ }^{3}$ University of California Observatories/Lick Observatory \\ Board of Studies in Astronomy and Astrophysics \\ University of California, Santa Cruz
}

Proceedings, Twelfth International Conference on Particles and Nuclei

Massachusetts Institute of Technology, June 1990

\section{PREPARED FOR THE U.S. DEPARTMENT OF ENERGY}

This report was prepared as an account of work sponsored by the United States Government. Neither the United States nor the United States Department of Energy, nor any of their employees, nor any of their contractors, subcontractors, or their employees, makes any warranty, express or implied, or assumes any legal liability or responsibility for the product or process disclosed, or represents that its use would not infringe privately-owned rights.

By acceptance of this article, the publisher and/or recipient acknowledges the U.S. Government's right to retain a nonexclusive, royalty-free license in and to any copyright covering this paper.

\section{DISCLAIMER}

This report was prepared as an account of work sponsored by an agency of the United States Government. Neither the United States Government nor any agency thereof, nor any of their employees, makes any warranty, express or implied, or assumes any legal liability or responsibility for the accuracy, completeness, or usefulness of any information, apparatus, product, or process disclosed, or represents that its use would not infringe privately owned rights. Reference herein to any specific commercial product, process, or service by trade name, trademark, manufacturer, or otherwise does not necessarily constitute or imply its endorsement, recommendation, or favoring by the United States Government or any agency thereof. The views and opinions of authors expressed herein do noi necessailiy state of reflect those of the United States Government or any agency thereof. 


\title{
NEUTRINO-INDUCED NUCLEOSYNTHESIS IN CORE-COLLAPSE SUPERNOVAE
}

\author{
D.H. Hartmann ${ }^{1}$, W.C. Haxton ${ }^{2}$, R.D. Hoffman $^{3}$, and S.E. Woosley ${ }^{1,3}$
}

${ }^{1}$ Physics Department, University of California, Lawrence Livermore National Laboratory'

${ }^{2}$ Nuclear Theory Group, Department of Physics, FM-15, University of Washington Seattle, Washington 98195

${ }^{3}$ University of California Observatories/Lick Observatory, Board of Studies in Astronomy and Ástrophysics, University of California, Santa Cruz

Almost all of the $3 \cdot 10^{53}$ ergs liberated in a core collapse supernova is radiated as neutrinos by the cooling neutron star. These neutrinos can excite nuclei in the mantle of the star by their neutral and charged current reactions. The resulting spallation reactions are an important nucleosynthesis mechanism that may be responsible for the galactic abundances of ${ }^{7} \mathrm{Li},{ }^{11} \mathrm{~B},{ }^{19} \mathrm{~F},{ }^{138} \mathrm{La},{ }^{180} \mathrm{Ta}$, and a number of other nuclei.

During a Type II supernova approximately $3 \cdot 10^{53}$ ergs is released by the cooling neutron star in the form of neutrinos of all flavors. ${ }^{1,2}$ These neutrinos can interact inelastically with the nuclei in the various ejected shells of the supernova via neutral or charged currents, often exciting particle unbound states that decay by neutron, proton, or $\alpha$-emission, e.g.

$$
(Z, A)+\nu \rightarrow(Z, A)^{*}+\nu^{\prime} \rightarrow\left\{\begin{array}{cc}
(Z, A-1) & +n \\
(Z-1, A-1) & +p \\
(Z-2, A-4) & +\alpha
\end{array}\right\}+\nu^{\prime}
$$

The higher temperature muon and tauon neutrinos, $\mathrm{T} \sim 8 \mathrm{MeV}$, are of particular importance because these neutrinos are capable of exciting both allowed and first-forbidden giant resonance states. ${ }^{3}$ This talk focuses on the important nucleosynthesis accompanying such reactions, which we call the neutrino process. ${ }^{4,5}$

While the mechanism of a core-collapse supernova is still poorly understood, theorists agree $^{1,2}$ that the total number of neutrinos and antineutrinos produced by the core collapse and subsequent cooling of a massive $\left(\sim 20 \mathrm{M}_{\odot}\right)$ siar is about ${ }^{8}$. The vast majnrity of these neutrinos are emitted as the neutron star cools: the neutrino luminosity after shock breakout at the neutrinosphere is well approximated by an exponential decay curve vith a time constant $\tau_{\nu} \sim 3 \mathrm{~s}$. The mean energy of the muon and tauon neutrinos is about 25 $\mathrm{MeV}$. Because charged current interactions lead to a larger $\nu_{e}-e$ scattering cross section, the electron neutrino distributions are cooler $(\mathrm{T} \sim 4 \mathrm{MeV})$.

Prior to the arrival of the shock wave (generated by the core bounce) a stellar zone at some fixed radius experiences an exponentially declining neutrino flux at approximately constant temperature and density. This is the pre-processing phase. As the shock wave passes 
through this zone the temperature rises to some peak value, and the necessary overpressure is generated to drive the zone outward in a rapid expansion. In the subsequent dynamic post-processing phase the temperature and density drop rapidly as a result of the adiabatic expansion of the zone, and the radial expansion leads to an additional reduction in the effective neutrino flux. We will see that important neutrino-induced nucleosynthesis occurs in both of these phases. The initial conditions encountered in the preprocessing phase are the end result of the star's evolution through hydrostatic burning. Our explorations were carried out for a "typical" Type II supernova progenitor of $20 \mathrm{M}_{\odot}{ }^{6,7}$ The specific model was that of Woosley and Weaver ${ }^{8}$, a Population I presupernova star that was evolved without mass loss, including semiconvection, and using the Caughlan et al. (1985) reaction rate for ${ }^{12} \mathrm{C}(\alpha, \gamma){ }^{16} \mathrm{O} .{ }^{9}$ The star begins its lifetime burning hydrogen, with an initial composition taken from Cameron except for an increase in helium mass fraction to 0.25 (and a corresponding decrease in the hydrogen mass fraction). After hydrogen burning has finished, the resulting ashes determine the initial composition at the onset of helium burning. This process continues, with the ashes of helium burning providing the starting composition for the carbon burning phase, etc. The result, just prior to core collapse, is a star with an iron core surrounded by layers of silicon, oxygen, neon, carbon, helium, and hydrogen (Fig. 1).

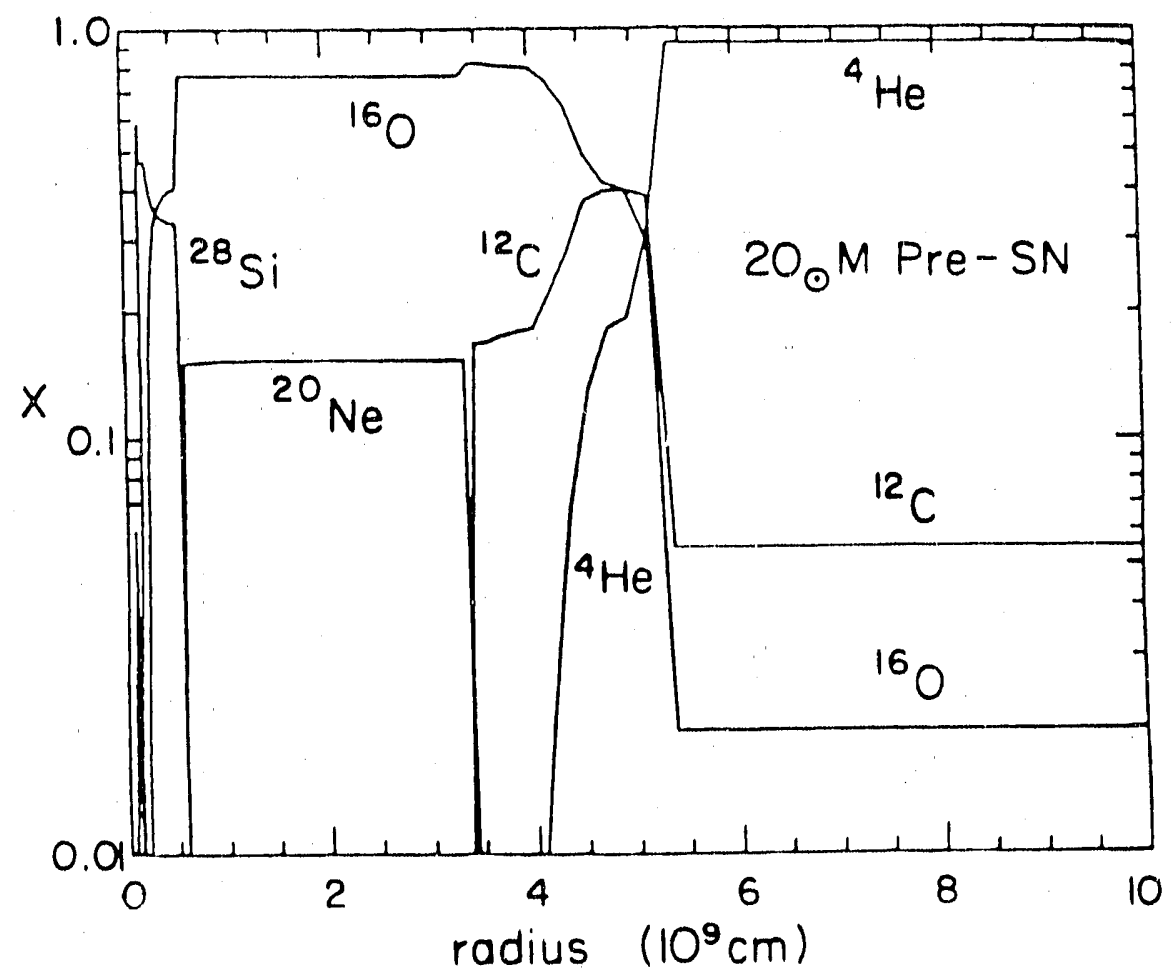

FIGURE 1

Mass fractions as a function of radius for the pre-supernova $20 \mathrm{M}_{\odot}$ star.

Inelastic neutral current scattering off nuclei is the principal reaction that generates the neutrino process. ${ }^{3}$ Although all neutrino flavors participate in this process, the higher 
temperature muon and tauon neutrinos are more important: the reactions of energetic neutrinos are favored by phase space, particularly in cases where nuclear thresholds are high. In addition, these neutrinos can strongly excite first-forbidden transitions to giant resonances which, as discussed below, dominate cross sections at intermediate energies. ${ }^{3}$

To fully incorporate neutrino physics in to the standard explosive nucleosynthesis reaction network, we required neutrino scattering cross sections and associated particle breakup branching ratios for approximately 300 light and medium mass nuclei. We began by studying a subset of 18 "node nuclei" that included most of the network species that accumulate appreciably: ${ }^{4} \mathrm{He},{ }^{8} \mathrm{Be},{ }^{12} \mathrm{C},{ }^{14} \mathrm{~N},{ }^{16} \mathrm{O},{ }^{20} \mathrm{Ne},{ }^{24} \mathrm{Mg},{ }^{28} \mathrm{Si},{ }^{32} \mathrm{~S},{ }^{36} \mathrm{Ar},{ }^{40} \mathrm{Ca},{ }^{44} \mathrm{Ti},{ }^{48} \mathrm{Cr},{ }^{52} \mathrm{Fe}$, ${ }^{56} \mathrm{Fe},{ }^{56} \mathrm{Ni},{ }^{60} \mathrm{Zn}$, and ${ }^{80} \mathrm{Zr}$. Detailed shell model studies were then undertaken to estimate the inclusive responses of these nuclei to supernova neutrinos. These results were then extrapolated to other network nuclei, using a scheme that took into account the dependencc of Gamow-Teller and first-forbidden cross sections on $A$ and $Z{ }^{5}$

The neutrino spectra were taken to be Fermi-Dirac distributions having temperatures ' and zero chemical potential

$$
n(E)=\frac{0.555}{T^{3}} \frac{E^{2}}{\epsilon^{E / T}+1}
$$

Calculations were performed for neutrino energies up to $150 \mathrm{MeV}$, so that accurate spectrumaveraged cross sections could be obtained for the highest temperatures of interest $(\mathrm{T} \sim 12$ $\mathrm{MeV}$ ).

The results are in accord with qualitative expectations. The closed-shell nuclei $\left({ }^{4} \mathrm{He},{ }^{16} \mathrm{O}\right.$, ${ }^{40} \mathrm{Ca}$ ) have smaller cross sections, a result of the weak GT strength and high thresholds for exciting isovector states. Because they are dominated by first-forbidden contributions, thesc cross sections have a steep $\mathrm{T}$ dependence. Nuclei in the middle of shells have substantial GT strength and thus the largest cross sections. At low $T$ these nuclei scatter neutrinos much more efficiently. However, the cross sections rise less steeply in $\mathrm{T}$ than in the case of the closed-shell nuclei. At $\mathrm{T} \sim 10 \mathrm{MeV}$ all large differences between nuclei have been smoothed out by the growing giant resonance response. Thus, at typical muon and taun neutrino temperatures, the cross sections/nucleon $\left(\sim 10^{-42} \mathrm{~cm}^{2}\right)$ are roughly independent of the nuclear species.

Branching ratios for the various decay channels of the excited nuclei were obtained from Hauser-Feshbach optical model calculations. Nuclear level densities were taken from a statistical formula, and the allowed decay channels were assumed to be $\mathrm{n}, \mathrm{p}, \alpha, \mathrm{d},{ }^{3} \mathrm{H},{ }^{3} \mathrm{H} c$, and $\gamma$. In most cases only the $\mathrm{n}, \mathrm{p}, \alpha$, and $\gamma$ channels are important. The code assumes isotopically pure nuclear states, and we neglected the small $(\sim 1 \%)$ isoscalar component in neutrino scattering.

The entire program described above was repeated for the charged current reactions $\left(\nu, e^{-}\right)$and $\left(\bar{\nu}, e^{+}\right)$. Note that the Fermi operator now contributes to allowed cross sections. Coulomb effects on the electron phase space and on the positions of giant resonances (in the Goldhaber-Teller model) were taken into account. 
Detailed results for neutrino process nucleosynthesis in each of the major supernova shells and during both the preprocessing and postprocessing phases are discussed in Ref. 5 . Some of the principai points can be summarized:

1) The net result of the neutrino process includes the instantaneous production of new isotopes, and also all of the subsequent nuclear reactions of the coproduced protons, neutrons, alphas, etc. In some cases the net effect of these reactions is to deritroy the product of interest; in others, these reactions may themselves produce new elements.

2) The products of the neutrino process may not survive the heating (and associated nuclear reactions) accompanying shock wave passage. Thus the effect of shock-wave heating is an important component of the calculations.

3) The post-processing phase is of particular importance for interior shells that expand off the neutron star at early times. Further shock-wave heating does not ocur. The composition of the shell during the neutrino illumination depends on the thermodynamic conditions extant in the hydrodynamically exparding and exponentially cooling material.

4) There are uncertainties in our calculations resulting from neutrino-nucleus cross sections, hadronic cross sections contributing to the network processing, the presupernova composition of the star, and the spectra and numbers of emitted neutrinos. We also anticipatc that some differences may arise when we extend our present work, specific to a particular presupernova progenitor ${ }^{8}$, to a wider range of pnssible parent stars.

Our calculations suggest abundances of ${ }^{7} \mathrm{~L},{ }^{11} \mathrm{~B},{ }^{19} \mathrm{~F},{ }^{138} \mathrm{La}$, and ${ }^{180} \mathrm{Ta}$ may be due almost entirely to the $\nu$-process. Significant : mounts of ${ }^{10} \mathrm{~B},{ }^{15} \mathrm{~N},{ }^{22} \mathrm{Na},{ }^{26} \mathrm{Al},{ }^{31} \mathrm{P},{ }^{35} \mathrm{Cl}$, ${ }^{39,40} \mathrm{~K},{ }^{51} \mathrm{~V},{ }^{45} \mathrm{Sc},{ }^{49} \mathrm{Ti},{ }^{50} \mathrm{~V},{ }^{55} \mathrm{Mn},{ }^{59} \mathrm{Co}$, and ${ }^{63} \mathrm{Co}$ are also produced so that, within the uncertainties of the model, these isotopes may also owe their origin predominantly to the: $\nu$-process. Below the production of ${ }^{7} \mathrm{Li},{ }^{11} \mathrm{~B}$, and ${ }^{19} \mathrm{~F}$ is discussed in more detail.

The only stable isotope of fluorine, ${ }^{19} \mathrm{~F}$, has a low solar abundance relative to its neighbor, ${ }^{20} \mathrm{Ne}$, of $1 / 3100$. Thus one can account for the entire observed abundance of ${ }^{19} \mathrm{~F}$ by producing; this isotope, in the same site where ${ }^{20} \mathrm{Ne}$ is synthesized, with a relative efficiency of only: $0.03 \%$.

The neutral current response for ${ }^{20} \mathrm{Ne}$ is dominated by states above the tirireshold for particle breakup. The optical model predictions for the ${ }^{20} \mathrm{Ne}\left(\nu, \nu^{\prime} n\right)^{19} \mathrm{Ne}$ and ${ }^{20} \mathrm{Ne}\left(\nu^{\prime}, \nu^{\prime} p\right)^{19} \mathrm{~F}$ branching ratios are $30 \%$ and $66 \%$ respectively. The neon rich shell, i.e., the region where carbon has at least partially burned but neon has not, lies between $(0.7-3.4) \cdot 10^{9} \mathrm{~cm}$ in our 20 $\mathrm{M}_{\odot}$ progenitor. For a distance of $2 \cdot 10^{9} \mathrm{~cm}$, a total energy in $\mu$ and $\tau$ neutrinos of $3 \cdot 10^{53}$ ergs, and an average neutrino temperature of $10 \mathrm{MeV}$, one finds that the total (instantaneous) production of mass-19 nuclei relative to ${ }^{20} \mathrm{Ne}$ parent nuclei is $0.3 \%$, or about an order of magnitude larger than the solar ratio. However, the survival of these nuclei at this point is still in doubt: both the nuclear processing of the protons and neutrons coproduced witl. the mass-19 nuclei and the nuclear heating when the shock wave (the ejection mechanism) passes can destroy these fragile nuclei. 
The network calculations show ${ }^{4}, 5$ that almost all of the ${ }^{19} \mathrm{Ne}$ is quickly destroyed, primarily by ${ }^{19} \mathrm{Ne}(n, \alpha)$. Thus this isotope does not survive to produce ${ }^{19} \mathrm{~F}$ by $\beta$ decay. Tho ${ }^{19} \mathrm{~F}$ can also be destroyed, with the key reaction being ${ }^{19} \mathrm{~F}(p, \alpha){ }^{16} \mathrm{O}$. However this process is the dominant proton reaction only if ${ }^{19} \mathrm{~F}$ is abundant. Otherwise ${ }^{23} \mathrm{Na}(p, \alpha){ }^{20} \mathrm{Ne}$ absorbs the protons. As the cross section for producing ${ }^{19} \mathrm{~F}$ is sharply dependent on $T_{\nu}$, the competition between these two reactions generates an interesting mechanism that prevents the overproduction of ${ }^{19} \mathrm{~F}$ and predicts a correlation between the ${ }^{19} \mathrm{~F}$ production and ${ }^{23} \mathrm{Na}$.

Table 1. Cross Sections, Fluences, and Nucleosynthesis of ${ }^{19} \mathrm{~F}$.

\begin{tabular}{lccccc}
\hline$T_{\nu}(\mathrm{MeV})$ & 4 & 6 & 8 & 10 & 12 \\
\hline${ }^{20} \mathrm{Ne}\left(\nu, \nu^{\prime} n\right)^{19} \mathrm{Ne}^{a}$ & 0.093 & 0.93 & 3.6 & 8.6 & 16.1 \\
${ }^{20} \mathrm{Ne}\left(\nu^{\prime}, \nu^{\prime} p\right)^{19} \mathrm{~F}^{a}$ & 0.25 & 2.2 & 7.9 & 18.6 & 34.4 \\
$\phi_{t o t}\left(\mathrm{~cm}^{-2}\right)^{b}$ & 2.96 & 1.97 & 1.48 & 1.18 & 0.99 \\
{$\left[{ }^{19} \mathrm{~F} /{ }^{20} \mathrm{Ne}\right]$} & 0.28 & 1.2 & 2.3 & 2.2 & 2.2 \\
{$\left[{ }^{19} \mathrm{~F} /{ }^{20} \mathrm{Ne}\right]_{f}$} & 0.14 & 0.6 & 1.2 & 1.1 & 1.1 \\
\hline
\end{tabular}

a Cross section in $10^{-42} \mathrm{~cm}^{2}$ (average for $\nu$ and $\bar{\nu}$ )

${ }^{6}$ Total fluence $\left(10^{38} / \mathrm{cm}^{2}\right)$ of $\mu$ and $\tau$ neutrinos and antineutrinos at $2 \times 10^{9} \mathrm{~cm}$

The second survival factor is the peak "explosion temperature" achieved when the shock wave passes through the ${ }^{20} \mathrm{Ne}$ shell. At a radius of $10^{9} \mathrm{~cm}$ the peak temperature is about $2.5 \cdot 10^{9} \mathrm{~K}$, while at $3 \cdot 10^{9} \mathrm{~cm}$ the value is lower, about $10^{9} \mathrm{~K}$. Fluorine is efficiently destroyed by ${ }^{19} \mathrm{~F}(\gamma, \alpha){ }^{15} \mathrm{~N}$ for temperatures $T \geq 1.7 \cdot 10^{9} \mathrm{~K}$. Thus only the ${ }^{19} \mathrm{~F}$ produced at large radii can survive the shock-wave heating.

The results of Woosley and $\mathrm{Haxton}^{4}$ for the production of ${ }^{19} \mathrm{~F}$ are summarized in Ta. ble 1. (A more careful calculation appears in Ref. 5.) The quantity $\left[{ }^{19} \mathrm{~F} /{ }^{20} \mathrm{Ne}\right]$ gives the ${ }^{19} \mathrm{~F} /{ }^{20} \mathrm{Ne}$ production ratio, normalized to the solar value, after all nuclear processing has been completed, in the absence of shock-wave heating. The quantity $\left[{ }^{19} \mathrm{~F} /{ }^{20} \mathrm{Ne}\right]_{f}$, the final ejected abindance ratio, gives the reduced value that results from including shock-ware heating.

For neutrino temperatures near the expected value $(\sim 8 \mathrm{MeV})$ the ejected ratio is rery' close to that observed in the sun. Assuming an uncertainty of a factor or two in the cosmic ${ }^{19} \mathrm{~F} /{ }^{20} \mathrm{Ne}$ abundance, we also see that temperatures much below $6 \mathrm{MeV}$ appear to be rulcd out, assuming that the $\nu$-process is the dominant nucleosynthesis mechanism for this element. Thus ${ }^{19} \mathrm{~F}$ serves as a crude supernova muon/tauon neutrino thermometer. Note that the growing importance of the reaction ${ }^{19} \mathrm{~F}(p, a){ }^{16} \mathrm{O}$ prevents the overproduction of ${ }^{19} \mathrm{~F}$ for $T_{1}>$ $8 \mathrm{MeV}$. The discovery of a plausible nucleosynthesis mechanism for ${ }^{19} \mathrm{~F}$ is a pleasing result. 
Another classic puzzle is presented by ${ }^{11} \mathrm{~B}$. Traditionally boron has been explained as a cosmic ray spallation product. However, ${ }^{11} \mathrm{~B}$ is underproduced relative to ${ }^{10} \mathrm{~B}$ in this process, leading to the suggestion that an (otherwise) undetected low-energy component in the cosmic ray flux could preferentially produce the heavier isotope. Similar calculations to those described above suggest that this contrived solution is unnecessary: the neutrino process production of ${ }^{11} \mathrm{~B}$ by ${ }^{12} \mathrm{C}\left(\nu, \nu^{\prime} n\right)^{11} \mathrm{C} \underset{\beta}{\longrightarrow}{ }^{11} \mathrm{~B}$ in the ${ }^{12} \mathrm{C}$ shell is very robust, fully accounting for the observed abundance of this isotope.

Perhaps the most exciting result is the production of ${ }^{7} \mathrm{Li}$, an isotope of great importance because of its role in the big bang. The standard big bang nucleosynthesis of this element, however, is not sufficient to account for the observed abundance. ${ }^{10}$ We find thit ${ }^{7} \mathrm{Li}$ (but very little ${ }^{6} \mathrm{Li}$ ) can be made from neutral current interactions with helium in two distinct stellar regions. Production in the helium shell, following spallation, occurs through ${ }^{3} \mathrm{H}(\alpha, \gamma)^{7} \mathrm{Li}$ and ${ }^{3} \mathrm{He}(\alpha, \gamma){ }^{7} \mathrm{Be}$. Under certain conditions (an $\alpha$-rich freeze out), it may also be produced during the post-processing phase, due to neutrino reactions with helium produced as the high-tem perature ejecta cools in nuclear statistical equilibrium. The total ${ }^{7} \mathrm{Li}$ production in our $20 \mathrm{M}_{\odot}$ model is about 0.6 of the observed abundance, which suggests that the $v$-process is responsible for most of the galactic ${ }^{7} \mathrm{Li}$.

\section{REFERENCES}

1) R.W. Mayle, Ph.D. thesis, Lawrence Livermore National Laboratory reprint UCRL53713 (1985); H.A. Bethe and J.R. Wilson, Ap. J. 295 (1985) 14.

2) J. Cooperstein, H.A. Bethe, and G.E. Brown, Nucl. Phys. 429 (1984) 527; E.A. Baron, J. Cooperstein, and S. Kahana, Phys. Rev. Lett. 55, (1985) 126 and Nucl Phys. A440 (1985) 744.

3) W.C. Haxton, Phys. Rev. Lett. 60 (1988) 1999.

4) S.E. Woosley and W.C. Haxton, Nature 334, (1988) 45.

5) S.E. Woosley, D. Hartmann, R.D. Hoffman, and W.C. Haxton, Ap. J. 356 (1990) 272.

6) W.D. Arnett and D.N. Schramm, Ap. J. Lett. 184 (1973) 247.

7) S.E. Woosley and T.A. Weaver, Ann, Rev, Astr. Astrop. 24 (1986) 205.

8) S.E. Woosley and T.A. Weaver, Phys. Rep. 163 (1988) 79.

9) G.R. Caughlan, W.A. Fowler, M.J. Harris, and B.A. Zimmerman, Atomic and Nuclear Data Tables 32 (1985) 197.

10) H. Reeves et al., Ap. J. 355 (1990) 18. 

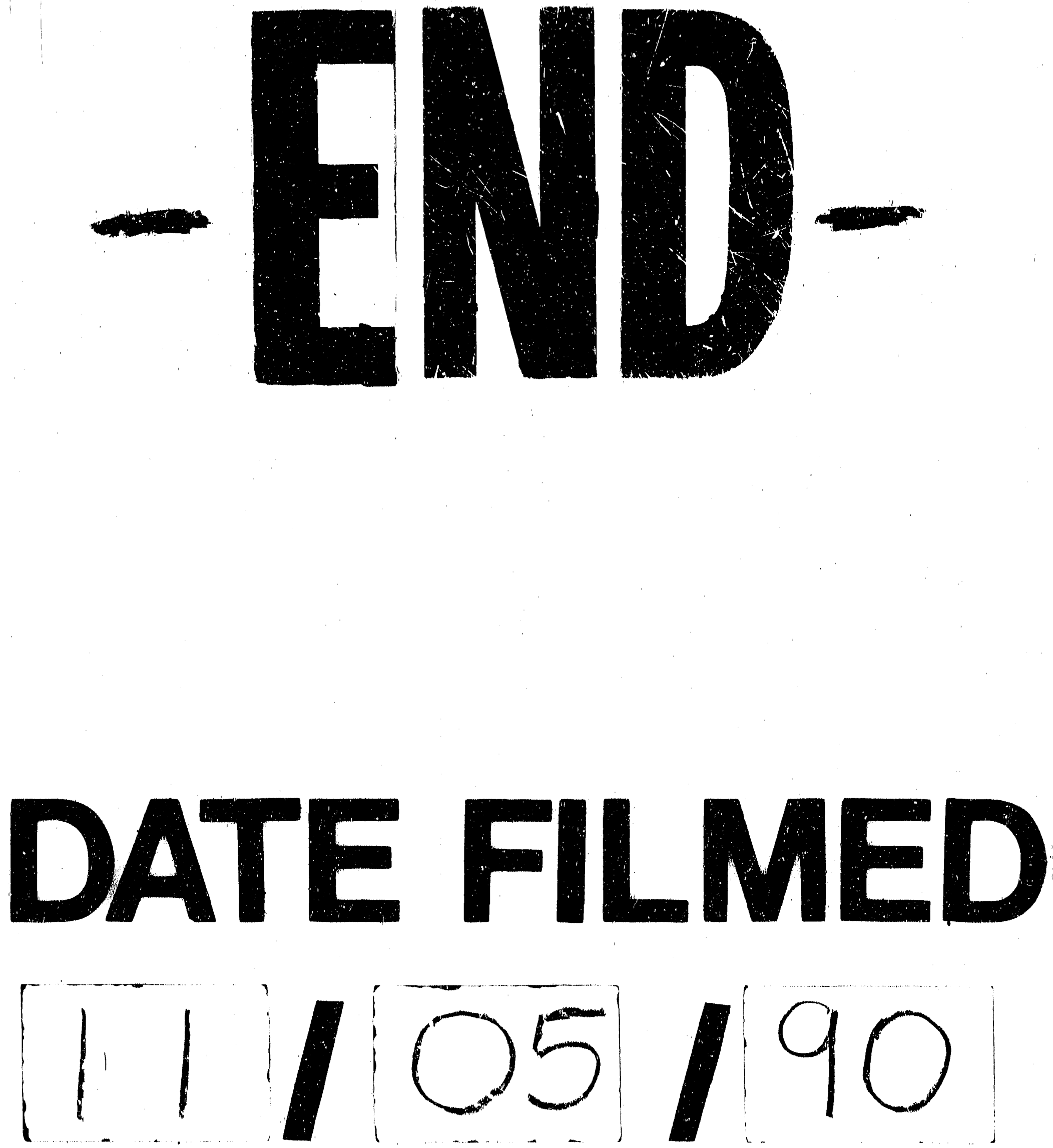
